\title{
Positron-annihilation-lifetime response and broadband dielectric relaxation spectroscopy: Diethyl phthalate
}

\author{
J. Bartoš, ${ }^{1}$ A. Alegría, ${ }^{2}$ O. Šauša, ${ }^{3}$ M. Tyagi, ${ }^{4}$ D. Gómez, ${ }^{2}$ J. Krištiak, ${ }^{3}$ and J. Colmenero ${ }^{2,4}$ \\ ${ }^{1}$ Polymer Institute of SAS, Dúbravská cesta 9, 84236 Bratislava, Slovakia \\ ${ }^{2}$ Departamento de Física de Materiales y Centro de Fisica de Materiales, Centro Mixto CSIC-UPV/EHU, Apdo. 1072, \\ 20080 San Sebastian, Spain \\ ${ }^{3}$ Institute of Physics of SAS, Dúbravská cesta 9, 84228 Bratislava, Slovakia \\ ${ }^{4}$ Donostia International Physics Center, 20018 San Sebastian, Spain
}

(Received 17 April 2007; published 12 September 2007)

\begin{abstract}
We report the results of a combined phenomenological analysis of the data from positron-annihilationlifetime spectroscopy (PALS) and the relaxation data from broadband dielectric spectroscopy (BDS) on diethyl phthalate (DEP). The ortho-positronium (o-Ps) lifetime, $\tau_{3}$, as a function of temperature over a temperature range from $67 \mathrm{~K}$ up to $300 \mathrm{~K}$ is compared with the spectral features and the relaxation parameters of the BDS spectra decomposed into the primary $\alpha$ and the secondary $\beta$ processes in the temperature range from $140 \mathrm{~K}$ up to $380 \mathrm{~K}$ by using the Williams-Watts scheme. Phenomenological model-free analysis of the $\tau_{3}$ - $T$ plot provides the three characteristic PALS temperatures, where the two most pronounced ones at $T_{g}^{\mathrm{PALS}}=185 \mathrm{~K}$ and $T_{b 2}$ $=245 \mathrm{~K}=1.32 T_{g}^{\mathrm{PALS}}$ are related to the glass-liquid transition and the onset of a quasiplateau region, respectively. In the case of a weaker bend effect at $T_{b 1}=210 \mathrm{~K}=1.14 T_{g}^{\mathrm{PALS}}$, a number of new coincidences with changes in the dielectric $\beta$ process have been found. They concern the changes in width parameter of the distribution function for the $\beta$ relaxation time and the activation energy of the $\beta_{\text {eff }}$ process, a crossover from the Arrhenius to the non-Arrhenius type of temperature dependence as well as with the onset of a short-time tail of the $\beta$ relaxation time distribution and finally, with changes in the relaxation strength of the $\alpha$ and $\beta_{\text {eff }}$ processes. All these findings indicate a close connection of the $o$-Ps annihilation parameters and relaxation characteristics of BDS response for the DEP matrix.
\end{abstract}

DOI: 10.1103/PhysRevE.76.031503

PACS number(s): 64.70.Pf, 78.70.Bj, 77.22.Gm, 61.66.Hq

\section{INTRODUCTION}

Structure-property relationships in condensed matter form continuing long-term topics of chemistry, physics, biology, and materials science. Traditionally, the former aspect of disordered materials is treated by means of diffraction methods by using $\mathrm{x}$ rays or neutron scattering techniques [1-4]. However, the relevant structural quantities, i.e., the static structure factors and the related radial distribution functions or the density fluctuations are not easily related in a simple and quantitative way to physical properties of practical significance such as dynamics and transport.

On the other hand, it is generally accepted that the thermodynamic, dynamic, and transport properties of amorphous or partially crystalline materials are closely related to the structural and dynamical disorder of the matrix. In this respect, the free volume concept received a large extension because of its conceptual simplicity and physical plausibility in interpretation of the above-mentioned behavior $[5,6]$. In spite of its large popularity, a sophisticated utilization of the free volume concept requires a firm establishing of some standard method for the free volume characterization. Here, positron-annihilation-lifetime spectroscopy (PALS) based on the annihilation of the bound system of positron and electron, the so-called ortho-positronium (o-Ps), is of great relevance. The $o$-Ps probe is a very sensitive indicator of the presence of local regions of the reduced electron density, such as vacancies in real crystals as well as free volume holes in amorphous materials [7-9]. The basic $o$-Ps annihilation parameter, i.e., $o$-Ps lifetime, $\tau_{3}$, is interpreted in free volume terms and is a measure of the size of these local free volume hole regions. Semiempirical models are used to characterize the PALS data in terms of free volume hole characteristics such as mean free volume hole size [10] and free volume hole fraction $[11,12]$. Subsequently, these free volume quantities are used in the correlation of various dynamics and transport phenomena [11-15].

As PALS experiment is carried out on a structurally and a dynamically fluctuating matrix with certain degrees of dynamical freedom determined by their chemical constitution and external variables such as temperature, $T$, and pressure, $p$, the PALS response is expected to be related to some parameters of the particle's mobility of glass-forming systems [16-20] ranging generally from picoseconds to seconds or even hours. Evidently, such a huge time scale of relaxation processes can be most effectively covered by broadband dielectric spectroscopy (BDS) [21-25].

Phenomenological model-free analysis of the $\tau_{3}-T$ dependences in a series of small molecular and polymeric glassformers revealed the existence of several characteristic PALS temperatures marked according to a unified notation [18] as $T_{g}^{\mathrm{PALS}}, T_{b 1}$ and $T_{b 2}=T_{r}[26]=T_{e}[27]=T_{k}$ [28] which are located at $T_{b 1} \sim 1.2-1.3 T_{g}^{\mathrm{PALS}}$ and $T_{b 2} \sim 1.4-1.7 T_{g}^{\mathrm{PALS}}[18]$. In order to utilize fully a potential of the PALS method for the characterization of any disordered system, the $\tau_{3}$ vs $T$ dependence and the origin of the PALS temperatures should be understood as completely as possible. Some progress has been achieved in this direction so far. Thus, it appears $[13,16-20,29]$ that $T_{b 2}$, marking the onset of quasiplateau region as temperature increases at relatively higher tempera- 
tures, coincides sometimes with the so-called Stickel's temperature $T_{B}^{\mathrm{ST}}[30]$ or/and the Schönhals temperature, $T_{B}^{\mathrm{SCH}}$ [31], at which significant changes in the relaxation parameters of the primary $\alpha$ process from dielectric spectroscopy (DS) occur. Moreover, at $T_{b 2}, \tau_{3}$, is often close to the mean relaxation time of the primary $\alpha$ process, $\tau_{\alpha}\left(T_{b 2}\right)$ as determined by standard peak analysis $[16,18,29]$. Slighter change in the slope at $T_{b 1}$ more or less close to the glass transition is sometimes observed $[16,18,29]$. It is also found that $T_{b 1}$ can be related to the temperature $T_{\alpha}(-6)$ at which the $\alpha$ relaxation time from DS reaches $10^{-6 \pm 1} \mathrm{~s}[18,20]$. Typically, the dielectric relaxation of usual glass-formers exhibits a broad spectrum with peak feature and a high frequency part from the so-called excess wing or/and secondary relaxation [21-24]. The correlation $T_{b 1} \sim T_{\alpha}(-6)$ seems to suggest that the motion associated with the high-frequency part of the dielectric relaxation spectrum could be in some way related to the change in the slope of the $\tau_{3}$ vs $T$ plot just at around $T_{b 1}$ as it is demonstrated very recently by some of us for glycerol [32].

In addition to the above-mentioned phenomenological PALS vs DS comparisons, theoretical [20] as well as modeling [20] approaches are also of relevance. Recently, some of us tried to explain the $T_{b 1}$ in diethyl phthalate (DEP) by using an extended free volume (EFV) model $[33,34]$. In this framework an agreement of the characteristic PALS temperature $T_{b 1}$ with the characteristic EFV temperature $T_{0}^{C G}$ for the PALS data suggests that the change in free volume microstructure as seen by the $o$-Ps probe could be related to the dynamic crossover in the primary $\alpha$ process from DS which occurs at $T_{0}^{C G}(\mathrm{DS}) \cong T_{0}^{C G}(\mathrm{PALS})$.

The aim of this paper is to present empirical analysis of the PALS data by using the dynamic ones from BDS on DEP. It is found that a combination of both experimental techniques can provide further insight into the dynamic nature of the PALS response. It is shown that such a joint phenomenological approach can contribute to more detailed explanation of the PALS response of the condensed system and the related characteristic PALS temperatures and subsequently, to deeper understanding of the liquid and supercooled liquid states of condensed matter.

\section{EXPERIMENTAL AND EVALUATION METHODS}

Material. Diethyl phthalate (DEP) $\quad \mathrm{C}_{6} \mathrm{H}_{4}(\mathrm{O}$ $\left.=\mathrm{C}-\mathrm{O}-\mathrm{CH}_{2}-\mathrm{CH}_{3}\right)_{2}$ from Aldrich Chemicals was used in both PALS and BDS studies. The glass transition temperature $T_{g}^{\mathrm{DSC}}=183 \mathrm{~K}$ and melting transition temperature $T_{m}^{\mathrm{DSC}}$ $=273 \stackrel{g}{\mathrm{~K}}$ on the basis of the differential scanning calorimetry (DSC) study [35].

\section{A. PALS}

The positron-annihilation-lifetime spectra were obtained by the conventional fast-fast coincidence method using plastic scintillators coupled to Phillips XP2020 photomultipliers. The time resolution of prompt spectra was about $320 \mathrm{ps}$. The activity of ${ }^{22} \mathrm{Na}$ source was $4 \mathrm{MBq}$. The radioactive positron ${ }^{22} \mathrm{Na}$ source plus samples assembly was kept in a rotary pump vacuum. During the low temperature measurements from $67 \mathrm{~K}$ up to $300 \mathrm{~K}$ samples in a holder were fixed at the end of a cold finger of a closed-cycle helium gas refrigerator Leybold with automatic temperature regulation. The temperature stability was about $1 \mathrm{~K}$ [36].

The positron-lifetime spectra were analyzed using the well-known PATFIT-88 software package [37] in terms of three annihilation modes: a short-term component from para-positronium $p$-Ps, $\tau_{1}$, an intermediate one attributed to "free" positron $\tau_{2}$, and a long-term one, related to free volume microstructure: ortho-positronium $o$-Ps, $\tau_{3}$.

The DEP sample was measured in three cycles: one during relatively slow cooling cycle giving the amorphous sample, while the remaining two during slow heating, one after rapid cooling, before the annihilation data acquisition, provided the structurally transformed material. The time of acquisition per one temperature point was at least 2 hours.

\section{B. BDS}

\section{Instrumental aspects}

Dielectric measurements on DEP above its $T_{g}$ were performed in a wide frequency range of $10 \mathrm{mHz}-20 \mathrm{GHz}$ using three different experimental setups depending upon the frequency range. In the frequency range of $10^{-2} \mathrm{~Hz}-10^{6} \mathrm{~Hz}$ dielectric measurements were made using a high-resolution Alpha dielectric analyzer supplied by Novocontrol, GmbH. For the frequency range of $10^{6} \mathrm{~Hz}-10^{9} \mathrm{~Hz}$, an Agilent impedance analyzer HP4291B was used. For both setups, samples were prepared between two gold plated electrodes of $30 \mathrm{~mm}$ and $10 \mathrm{~mm}$ diameters, respectively. Teflon spacers of $0.1 \mathrm{~mm}$ thickness and negligible area were used to maintain the constant distance between the two electrodes. For both setups, isothermal frequency measurements were carried out on a series of temperatures with temperature stability better than $0.05 \mathrm{~K}$. Measurements at and above room temperature were also carried out in the frequency range of $200 \mathrm{MHz}-20 \mathrm{GHz}$ using an integrated system of HP8361A vector network analyzer (VNA) and dielectric probe kit HP85070E with an open ended coaxial (OEC) probe. Complex permittivities were recorded using software supplied with a dielectric probe kit which controls the vector analyzer for dielectric measurements. More details about the setups, related accuracies, and calibration of the instruments can be found elsewhere [25]. Below $T_{g}$, measurements were made in the frequency range of $10 \mathrm{mHz}-10^{6} \mathrm{~Hz}$ using Alpha analyzer only.

\section{Evaluation aspects}

The treatment of the dielectric data is done assuming the picture originally developed by William and Watts [38]. In this picture, one considers that the total dielectric relaxation is driven by two separate mechanisms. The fast one, usually, the $\beta$ relaxation, is attributed to the restricted motions in an otherwise rigid environment. This mechanism is the dominant one below glass transition temperature. However, only above $T_{g}$ where primary $(\alpha)$ relaxation takes the dominant position, the total relaxation of the dipolar unit is achieved. 
In this approach, the total correlation function can be written as

$$
\varphi(t)=\varphi_{\alpha}(t)\left[f_{\alpha}+\left(1-f_{\alpha}\right) \varphi_{\beta}(t)\right],
$$

where $\varphi_{\alpha}(t)$ and $\varphi_{\beta}(t)$ are the two normalized relaxation functions corresponding to primary and secondary processes, respectively. In Eq. (1), $\left(1-f_{\alpha}\right)$ is the fraction of the whole relaxation that is achievable by the secondary process. It can also be seen easily that when the two processes are well separated, i.e., when $\varphi_{\alpha}(t)$ decay is not significant until the $\varphi_{\beta}(t)$ is complete $\left(\varphi_{\alpha} \sim 1\right.$ when $\left.\varphi_{\beta} \sim 0\right)$, the above equation (1) can be approximated as the simple summation

$$
\varphi(t)=f_{\alpha} \varphi_{\alpha}(t)+\left(1-f_{\alpha}\right) \varphi_{\beta}(t) .
$$

Equation (2), which is a special case of Eq. (1), has been used extensively in literature to analyze the dielectric data by assuming simple superposition of the two relaxation processes. For the sake of clarity, in this paper, we will refer to Eq. (1) as William-Watts ansatz, while the second Eq. (2) will be called the additive ansatz as it is simple superposition of the two observed responses.

In a typical dielectric experiment, the observed frequency dependent complex permittivity $\varepsilon^{*}(\omega)$ can be expressed as

$$
\varepsilon^{*}(\omega)-\varepsilon_{\infty}=\left(\varepsilon_{0}-\varepsilon_{\infty}\right) \Phi^{*}(\omega),
$$

where $\Phi^{*}(\omega)$ is the normalized relaxation function and $\varepsilon_{0}$ and $\varepsilon_{\infty}$ are the two limiting values of a dielectric constant. As mentioned above, when the two processes are well separated, the WW ansatz turns into additive ansatz and $\Phi^{*}(\omega) \Phi^{*}$ can be written as

$$
\Phi^{*}(\omega)=\left[\varepsilon^{*}(\omega)-\varepsilon_{\infty}\right] /\left(\varepsilon_{0}-\varepsilon_{\infty}\right)=f_{\alpha} \Phi_{\alpha}^{*}(\omega)+\left(1-f_{\alpha}\right) \Phi_{\beta}^{*}(\omega),
$$

where $\Phi_{\alpha}^{*}(\omega)$ and $\Phi_{\beta}^{*}(\omega)$ are the normalized relaxation functions in the frequency domain corresponding to the primary and secondary relaxations, respectively. In this case, the total dielectric strength $\Delta \varepsilon$ is just the summation of individual dielectric strengths. Now, to describe the dielectric spectrum shape, it is well known that the dielectric shape of primary and secondary relaxation processes is non-Debye. However, this non-Debye shape can be accounted in terms of distribution functions of relaxation times, namely, $g_{\alpha}\left(\log _{10} \tau\right)$ and $g_{\beta}\left(\log _{10} \tau\right)$, which are usually temperature dependent with characteristic relaxation times $\tau_{\alpha}(T)$ and $\tau_{\beta}(T)$, respectively. Below the glass transition temperature, where secondary relaxation can be observed without the influence of primary relaxation, usually, $\tau_{\beta}(T)$ follows an Arrhenius-like temperature dependence as

$$
\tau_{\beta}(T)=\tau_{\beta 0} \exp \left(E_{0} / k_{B} T\right)
$$

where $E_{0}$ represents an average activation energy of the $\beta$ process and $k_{B}$ is the Boltzmann's constant. Within this approach, the distribution of relaxation times can be interpreted in terms of a distribution of activation energies. Usually, a Gaussian distribution of energies provides a reasonable description of the secondary relaxation below $T_{g}$. However, in a temperature range where the two relaxations start to overlap, the temperature dependence of $\tau_{\beta}$ can be different than the

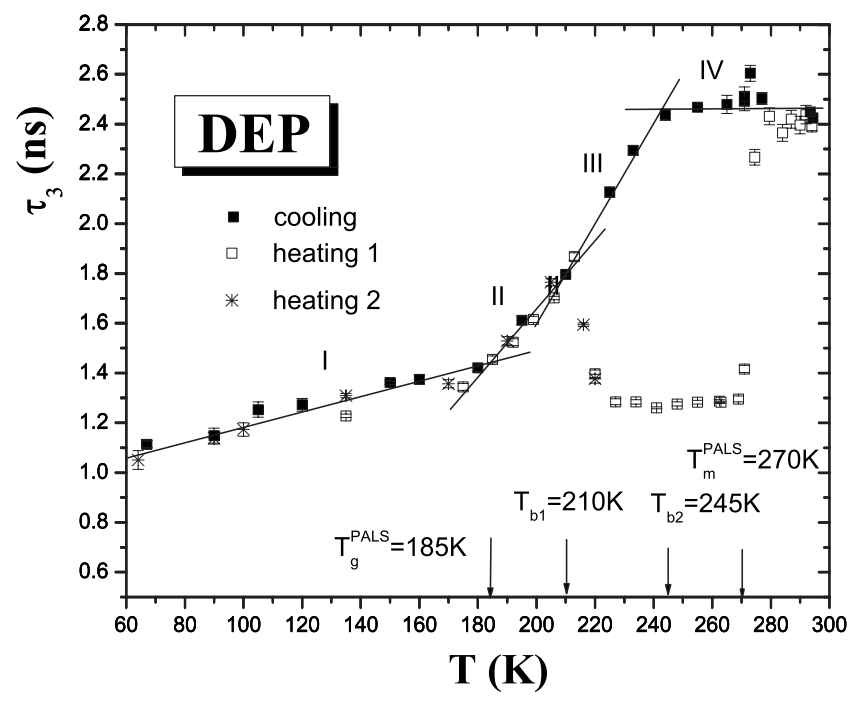

FIG. 1. Ortho-positronium lifetime, $\tau_{3}$, as a function of temperature as obtained in cooling (black squares) and two heating cycles (empty squares and crosses) with different heating rates. The first measurement was performed in a heating cycle from the low temperature region up to room temperature after rapid cooling of the sample which provided the structurally transformed material (empty squares). The next measurement was carried out during a relatively slow cooling cycle from room temperature down to low temperature giving the amorphous sample (solid squares). Finally, the cooled sample was measured in another heating cycle with different temperature steps as previously which provided the structurally transformed material again (crosses). The characteristic PALS temperatures $T_{g}^{\mathrm{PALS}}, T_{b 1}$, and $T_{b 2}$ from the best linear regression analyses are marked. The error bars are given and in most cases are smaller than the data points.

one that is extrapolated from low temperatures. This situation is in fact obtained using Eq. (1) which can be split in summation of the two terms as

$$
\varphi(t)=f_{\alpha} \varphi_{\alpha}(t)+\left(1-f_{\alpha}\right) \varphi_{\alpha}(t) \varphi_{\beta}(t),
$$

where the second term would correspond to the effective $\beta$ process, $\varphi_{\beta \text { eff }}(t)=\varphi_{\alpha}(t) \varphi_{\beta}(t)$. In our approach, $\tau_{\beta}$ and the shape of the $\alpha$ process are fixed from the temperature regions where the respective parameters can be obtained from well separated processes. This approach has been applied to a number of different glass formers to describe the dynamics over broad range of temperatures [21,23,39-41]. In addition this approach involves two parameters less than the additive ansatz approach [Eq. (2)] and therefore, appears to be more appropriate to the current study, since the uncertainty of the parameters so obtained is significantly reduced.

\section{RESULTS}

\section{A. PALS results}

As mentioned in Sec. II, the DEP sample was measured in both cooling and heating cycles. Figure 1 shows the $o$-Ps lifetime, $\tau_{3}$, as a function of temperature for DEP from $67 \mathrm{~K}$ up to $300 \mathrm{~K}$ as measured in these various cycles. The $o$-Ps 
data are practically independent of the cycle type from glassy state up to $210 \mathrm{~K}$. Above this temperature, the two slow heating cycles indicate certain structural change which is attributed to the transformation from supercooled liquid to a crystalline solid state as supported by comparison with the DSC data from the literature [35]. Therefore, the PALS results are discussed in two separate ways. The PALS response regarding the amorphous type sample is analyzed in detail here. The PALS one concerning the structurally transforming material will be discussed later.

Phenomenological, i.e., microscopic model-free, analysis of the $\tau_{3}-T$ dependence by using the best linear regression fitting $[16,18-20,29]$ indicates the presence of four regions of different thermal behavior. These regions can be approximated by the following linear expressions, where $r$ is a regression coefficient:

Region I from $67 \mathrm{~K}$ up to $180 \mathrm{~K}$,

$$
\tau_{3}=(2.67 \pm 0.44) \times 10^{-3} T+(0.92 \pm 0.06), \quad r=0.925 ;
$$

region II from $186 \mathrm{~K}$ up to $206 \mathrm{~K}$,

$$
\tau_{3}=(1.21 \pm 0.22) \times 10^{-2} T-(0.77 \pm 0.44), \quad r=0.940 ;
$$

region III from $213 \mathrm{~K}$ up to $233 \mathrm{~K}$,

$$
\tau_{3}=(2.16 \pm 0.02) \times 10^{-2} T-(2.72 \pm 0.05), \quad r=0.999 .
$$

Finally, in region IV above $245 \mathrm{~K}$ up to $300 \mathrm{~K}$, the average value of the $o$-Ps lifetime is quasiconstant and reaches $(2.50+/-0.09) \mathrm{ns}$. The intersection points of these linear expressions define the characteristic PALS temperatures, $T_{g}^{\mathrm{PALS}}=185 \mathrm{~K}, T_{b 1}=210 \mathrm{~K}$, and finally, $T_{b 2}=245 K$ [33].

\section{B. BDS results}

Figures 2(a)-2(h) display the representative BDS spectra of DEP over a very wide frequency range covering the whole temperature range from $140 \mathrm{~K}$ up to $298 \mathrm{~K}$ together with the results of their phenomenological spectral analyses. In general, the BDS spectra of DEP exhibit the typical features known for many small molecular and polymer glass formers [24]. Starting from below, at $150 \mathrm{~K}$, lying below the $T_{g}^{\text {PALS }}$ $=185 \mathrm{~K} \sim T_{g}^{\mathrm{DSC}}=183 \mathrm{~K}$, the BDS spectrum consists of the small dielectric loss peak which is attributed to the secondary $\beta$ process. This rather symmetric peak can be approximated by the log-normal distribution function $G\left(\log _{10} \tau_{\beta}\right)$ implying the Gaussian distribution of activation energies $G\left(E_{\beta}\right)$ as described in the data evaluation section above. On crossing the $T_{g}^{\text {PALS }}$, a strong main peak feature with the so-called highfrequency wing comes into the dielectric frequency window used. The BDS spectra above $T_{g}$ can be considered as being composed of both the primary $\alpha$ relaxation and the secondary $\beta$ process. Subsequently, for more detailed spectral analysis the Williams-Watts (WW) ansatz can be applied. The results of such analysis are also presented in Figs. 2(b)-2(g). Example of the BDS spectra at around $T_{g}^{\mathrm{PALS}}$ and that for a deeply supercooled liquid DEP at $195 \mathrm{~K}$ lying in between $T_{g}^{\mathrm{PALS}}$ and $T_{b 1}$ are given in Figs. 2(b) and 2(c), where the $\alpha$ process dominates over the weaker $\beta_{\text {eff }}$ one. On increasing the temperature, the secondary $\beta_{\text {eff }}$ process intensifies as it can be seen at the first characteristic PALS temperature $T_{b 1}=210 \mathrm{~K}$ and $T_{b 1}<T=220 \mathrm{~K}<T_{b 2}$ in Figs. 2(d) and 2(e), respectively. At $T=233 \mathrm{~K}$ lying still below $T_{b 2}$ the $\beta_{\text {eff }}$ process becomes more effective in the dielectric relaxation-Fig. 2(f). At still higher temperature, e.g., $245 \mathrm{~K}$ only one peak feature is present in the BDS spectra which is even more evident at very high temperatures, e.g., $T$ $=298 \mathrm{~K}$-Fig. 2(h).

Figure 3 shows the relaxation map of the relaxation times vs inverse of the temperature for all the motional processes observed in DEP in our BDS study. The primary $\alpha$ relaxation has been measured over a wide temperature range in the liquid state above $T_{g}^{\mathrm{DSC}}=183 \mathrm{~K}$ up to $380 \mathrm{~K}$. At relatively lower temperatures up to ca. $270 \mathrm{~K}$ the relaxation times exhibit the non-Arrhenius character which is followed by an approximately linear course above $270 \mathrm{~K}$ suggesting a crossover to the Arrhenius-type dependence in the relatively high temperature region. The secondary process detected over the temperature range from $140 \mathrm{~K}$ up to ca. $245 \mathrm{~K}$ shows also a complicated course reflecting a change in the character of the local scale mobility with temperature. At low temperatures, below $T_{g}^{\mathrm{PALS}}=185 \mathrm{~K}$ the usual Arrhenius-type temperature dependence of the $\beta$-relaxation times is evident as it is observed very often for glass formers. On crossing the $T_{g}^{\mathrm{PALS}}$, the Arrhenius character persists up to $T_{\mathrm{cr}} \sim 215 \mathrm{~K}$. Above $215 \mathrm{~K}$ a clear crossover from the Arrhenius type to the nonArrhenius-type dependence occurs. Note, however, that in the fitting procedure it was assumed that the Arrhenius $T$ dependence of the $\beta$ relaxation remains the same as at low temperatures whereas the crossover to the non-Arrhenius behavior of the effective $\beta$ process is properly captured by the WW ansatz. Similar crossover behaviors have been found for both small molecules $[39,40]$ as well as polymer $[21,23,41]$ glass-forming systems. Finally, on further increasing the temperature the apparent dominance of the $\beta_{\text {eff }}$ process over the $\alpha$ relaxation appears at around $245 \mathrm{~K}$.

Figure 4 shows the relaxation strengths of the $\alpha$ process, $\Delta \varepsilon_{\alpha}$, and the $\beta_{\text {eff }}$ relaxation, $\Delta \varepsilon_{\beta \text { eff }}$, as well as that of the total relaxation process, $\Delta \varepsilon$. The change in temperature dependence of $\Delta \varepsilon_{\alpha}$ and $\Delta \varepsilon_{\beta \text { eff }}$ occurs above $T_{\mathrm{cr}}=215 \mathrm{~K}$. On further increasing the temperature the $\beta_{\text {eff }}$ contribution becomes the most effective in the dielectric relaxation process. Note that already at these temperatures the two relaxation components $\alpha$ and $\beta_{\text {eff }}$ are looking very much similar in all the characteristics [see Fig. 2(f)]: shapes, intensity, and peak position. This makes any separation procedure rather uncertain. Finally, above $\sim 245 \mathrm{~K}$ the total relaxation is fully dominated by a single process.

The so-called Schönhals plot [31] for the total relaxation strength as a function of the logarithm of relaxation frequency is presented in Fig. 5. Three regions of different behavior can be seen whose linear representations determine the intersection points which define the characteristic BDS temperatures: $T_{B 1}^{\mathrm{SCH}}=212 \mathrm{~K}$ and $T_{B 2}^{\mathrm{SCH}}=235 \mathrm{~K}$. These temperatures reveal uncompensated changes in $\tau_{\alpha}$ and/or $\Delta \varepsilon$. 

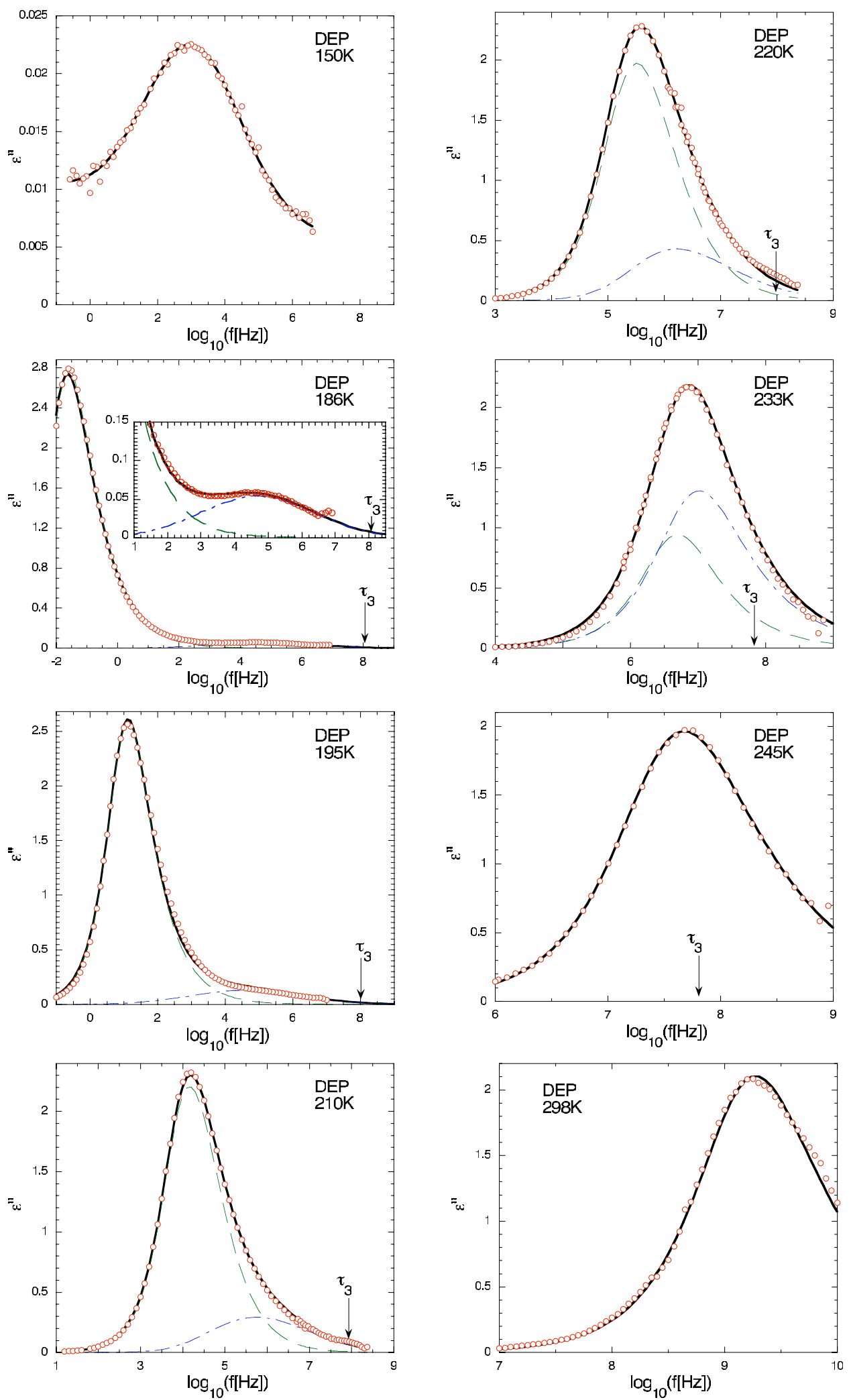

FIG. 2. (Color online) Dielectric loss spectra of DEP decomposed by using the WW ansatz into the $\alpha-(---)$ and $\beta_{\mathrm{eff}^{-}}$(-.-.-) relaxation component at selected temperatures: (a) in supercooled glassy state at $150 \mathrm{~K}$; (b) at $T \sim T_{g}$; (c) in strongly supercooled liquid state at $T_{g}$ $<195 \mathrm{~K}<T_{b 1}$; (d) at $T_{b 1}=210 \mathrm{~K}$; (e) in weakly supercooled liquid at $T_{b 1}<220 \mathrm{~K}$ or (f) $233 \mathrm{~K}<T_{b 2}$, respectively; (g) at $T_{b 2}=245 \mathrm{~K}$ and finally, (h) in normal liquid state at $298 \mathrm{~K}$. The arrows mark the equivalent frequencies, $f_{3}$, related to the $o$-Ps lifetimes via $f_{3}(T)$ $=1 / 2 \pi \tau_{3}(T)[32]$ which indicate how the secondary $\beta$ - and primary $\alpha$-relaxation processes reach the ns scale of the PALS method-see text. 


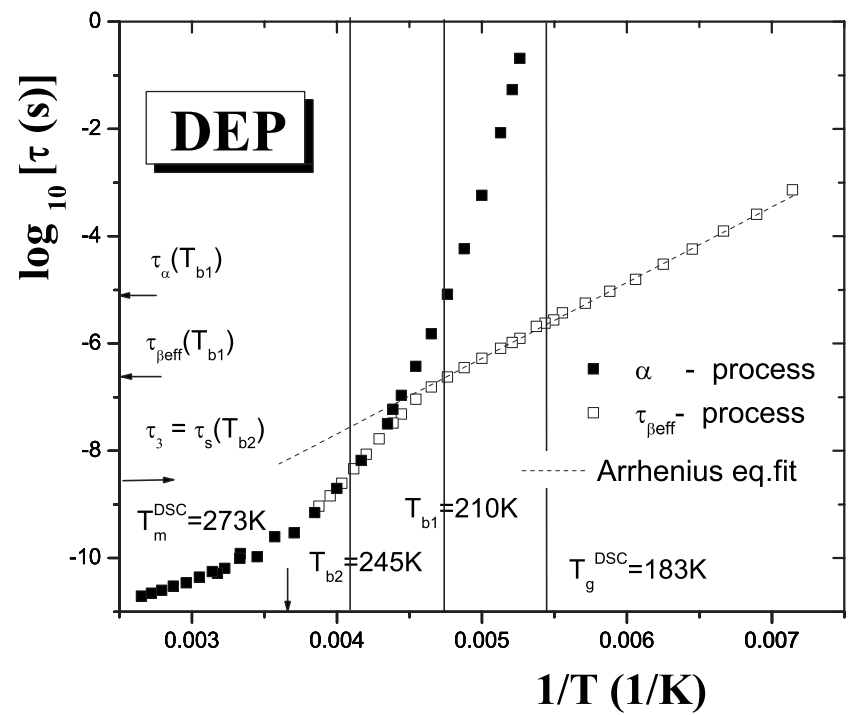

FIG. 3. Arrhenius representation of the relaxation times of the main process (solid squares) and the effective $\beta_{\text {eff }}$ process (empty squares). The $o$-Ps lifetime value $\tau_{3}$ at $T_{b 2}$ and the $\alpha$ - and

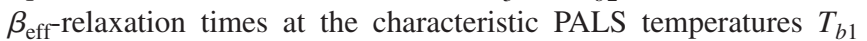
and $T_{b 2}$ are also given.

\section{DISCUSSION}

\section{Phenomenological analysis of PALS and BDS data}

In order to reveal further the nature of the PALS response and the physical meaning of the characteristic PALS temperatures, we discuss the PALS data by using the detailed BDS results. At first glance, the PALS response exhibits roughly a quasisigmoid form which is the typical feature of most of the glass-forming systems when measured over a very wide temperature range from a glassy state through a

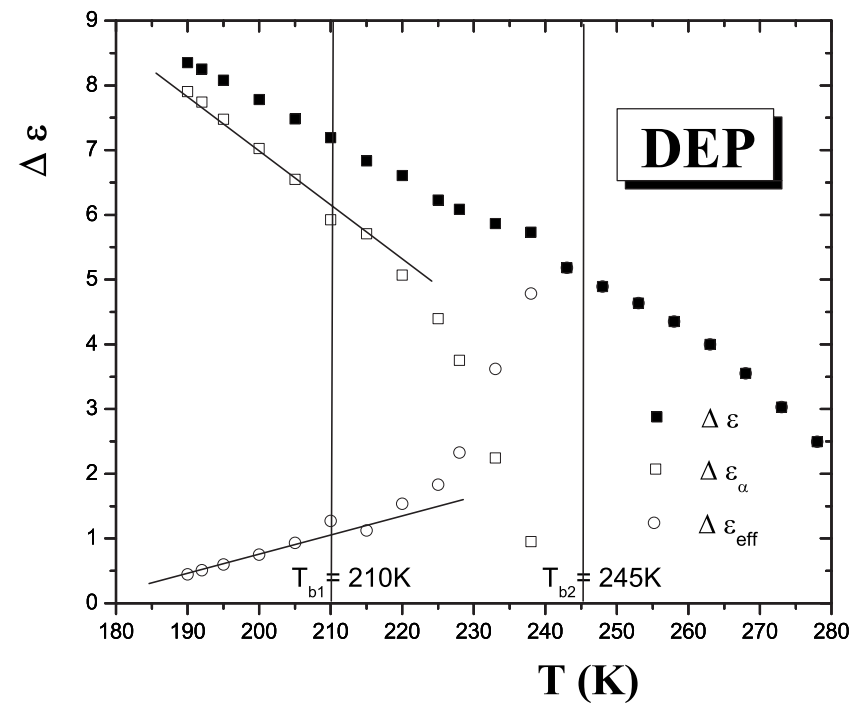

FIG. 4. Relaxation strength of the total relaxation process $\Delta \varepsilon$ (solid squares), as well as of the individual $\alpha$ - (empty squares) and $\beta_{\text {eff- }}$ (empty circles) relaxation components as obtained from the WW ansatz as a function of temperature.

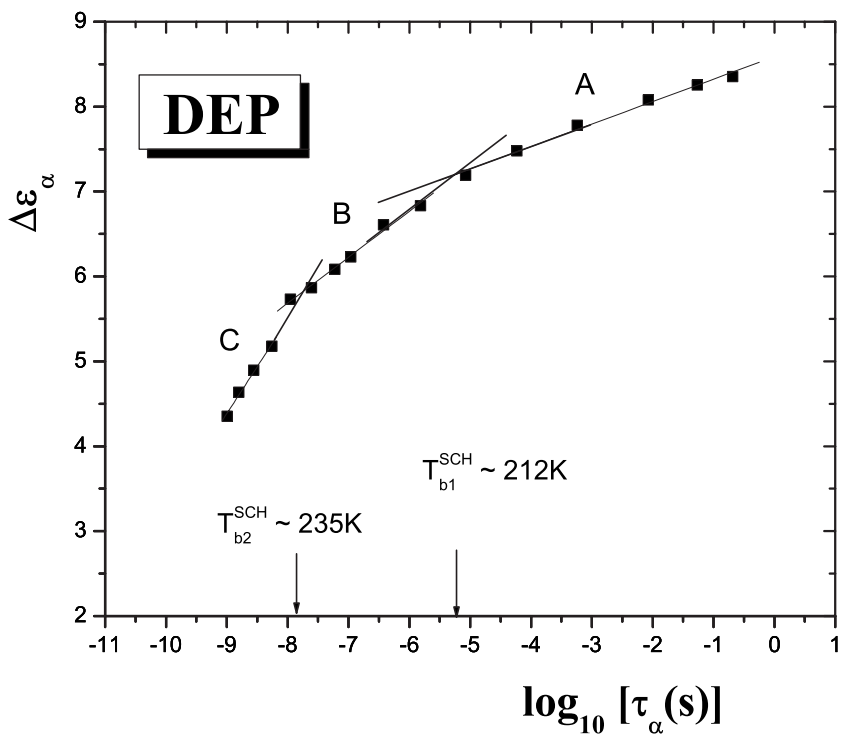

FIG. 5. Schönhals plot for the main relaxation process indicating the presence of the two characteristic BDS temperatures $T_{B 1}^{\mathrm{SCH}}$ $=212 \mathrm{~K}$ and $T_{B 2}^{\mathrm{SCH}}=235 \mathrm{~K}$.

supercooled liquid state up to a normal liquid [7-9,18,26-29]. Approximate linear-type regression analysis of $\tau_{3}-T$ dependence suggests the three characteristic PALS temperatures in the temperature range from $67 \mathrm{~K}$ up to $295 \mathrm{~K}$ with the following values of $o$-Ps lifetime: $\tau_{3}\left(T_{g}^{\text {PALS }}\right)$ $=1.41 \mathrm{~ns}, \tau_{3}\left(T_{b 1}\right)=1.77 \mathrm{~ns}$, and finally, $\tau_{3}\left(T_{b 2}\right) \sim 2.50 \mathrm{~ns}$. The last two values are shown also in Fig. 3 and they can be compared with the temperature dependences of the corresponding relaxation times for all the relaxation processes. Starting from above, we can see that at $T_{b 2}=245 \mathrm{~K}$ the plateau value of $o$-Ps lifetime is comparable with the relaxation time from the single relaxation-Fig. $2(\mathrm{~g})$. This finding for DEP is fully compatible with those for several other systems from our recent combined PALS and DS investigations on small molecular [16,18-20,32] as well as polymer [18] glass formers suggesting the approximate equality of the plateau $o$-Ps lifetime with the $\alpha$ relaxation time at this temperature. Note that these previous findings $\tau_{3}\left(T_{b 2}\right) \cong \tau_{\alpha}\left(T_{b 2}\right)$ have been based on comparisons of the plateau $\tau_{3}$ value with the peak relaxation time from standard peak analyses being attributed to the $\alpha$ process or to the so-called unified $\alpha \beta$ process without any detailed spectral analysis. One interesting difference within the class of crystallizing small molecular glass formers such as glycerol (GL) [15] and orthoterphenyl (OTP) [42] and DEP can be mentioned. In GL, which is of the intermediate fragility, the plateau effect occurs at $T_{b 2}$ which is commensurate with the corresponding melting point, $T_{m}$ [19]. In contrast, for fragile DEP the $\tau_{3}\left(T_{b 2}\right) \cong \tau_{\text {single }}\left(T_{b 2}\right)$ equality is reached already at about $30 \mathrm{~K}$ below the corresponding $T_{m}^{\mathrm{DSC}}=273 \mathrm{~K}$. As similar situation has been found for further fragile OTP systems but this finding has not been discussed [42].

On decreasing the temperature, the $o$-Ps lifetime values remain within the nanosecond range, while the relaxation times become considerably higher due to a dramatic slowing down of the main relaxation process. At $T_{b 1}$, the $\alpha$ relaxation 


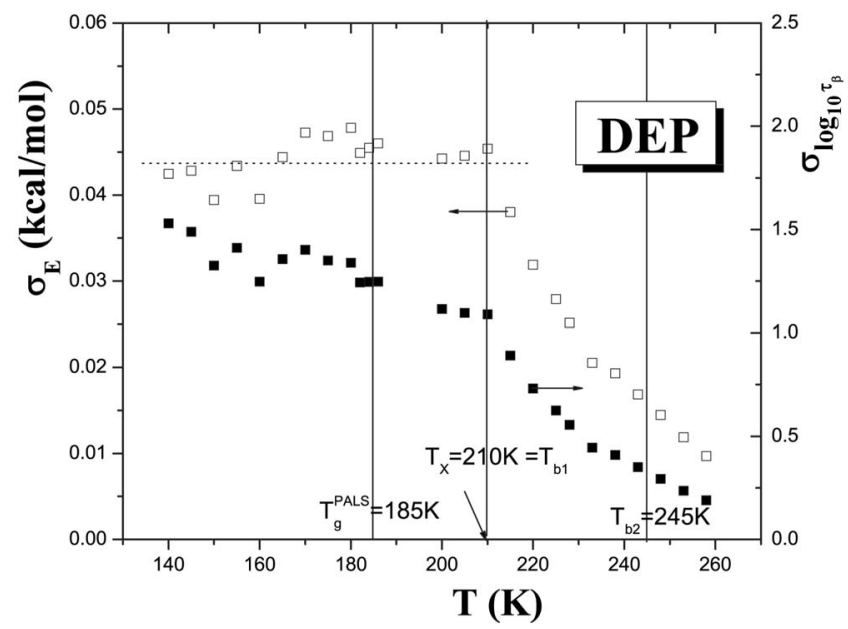

FIG. 6. Width parameters of the Gaussian distribution function for activation energy, $\sigma_{E_{\beta}}$, and the corresponding log-normal distribution for the $\beta$-relaxation time $\sigma_{\log _{10}} \tau_{\beta}$ as a function of temperature. Pronounced changes above $T_{x}=210 \mathrm{~K}$ close to the characteristic PALS temperature $T_{b 1}$ are evident.

time reaches the value $\tau_{\alpha}\left(T_{b 1}\right)=10^{-5.1} \mathrm{~s}$ which is consistent with our very recent empirical finding, $\tau_{\alpha}\left(T_{b 1}\right)=10^{-(6 \pm 1)} \mathrm{s}$ $[18,20]$. Thus, some universal feature of the main loss process seems to be related to a weaker bend effect in the PALS response. Likely, because of the three orders of magnitude difference between $\tau_{3}$ and $\tau_{\alpha}$ this feature could be related to the high-frequency part of the dielectric process which in framework of the WW analysis belongs to the secondary $\beta_{\text {eff }}$ relaxation-Fig. 2.

Indeed, as it follows from comparison of Fig. 1 with Figs. 3 and 4 the characteristic PALS temperature $T_{b 1}$ lies quite close to the crossover temperature, $T_{\mathrm{cr}}$, at which both the time and relaxation strength parameters of the secondary $\beta_{\text {eff }}$ process exhibit the significant variations. In particular, the relaxation time of the $\beta_{\text {eff }}$ process changes its character from the Arrhenius type in the glassy state with a continuation over $\sim 25 \mathrm{~K}$ into the deeply supercooled liquid one to the non-Arrhenius one in the slightly supercooled liquid state at $T_{\text {cr }} \sim 215 \mathrm{~K}$. At the same time, the relaxation strength of the $\alpha$ process starts to reduce and that of the $\beta_{\text {eff }}$ contribution becomes more effective in the dielectric relaxation. However, at higher temperatures $(T>240 \mathrm{~K})$ the separation of the two relaxation components is questionable because both exhibit similar characteristics. Thus, above the total relaxation can be considered as a single process.

In spite of the existence of the above-mentioned temperature matching between the changes in the $o$-Ps lifetime and the crossovers in the $\beta_{\text {eff }}$ relaxation time and the $\beta_{\text {eff }}$ relaxation strength, the two-order difference between the time scales for the $o$-Ps annihilation lifetime and the mean $\beta_{\text {eff }}$ process still does exist. In particular, at $T_{b 1} \tau_{3}=1.8 \times 10^{-9} \mathrm{~s}$ $<\tau_{\beta \text { eff }}=2.4 \times 10^{-7} \mathrm{~s}$. This fact calls for a more detailed inspection of both the primary $\alpha$ and especially, of the secondary $\beta$ process as presented in Figs. 2, 6, and 7. Figure 2(b) shows that the high-frequency tail of the secondary $\beta$ peak reaches the ns scale of PALS method just at around $T_{g}^{\text {PALS }}$. This finding correlates with increasing trend in the $o$-Ps life-

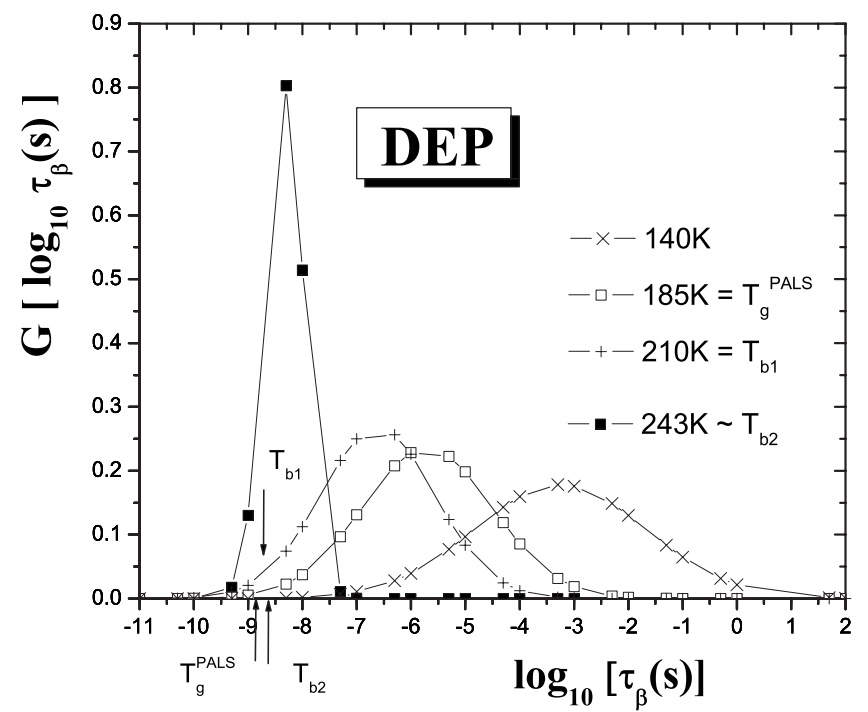

FIG. 7. Distributions of the $\beta$-relaxation times at several selected temperatures at $140 \mathrm{~K}$ in the glassy state, $T_{g}^{\text {PALS }}=185 \mathrm{~K}$, $T_{b 1}=210 \mathrm{~K}$ in the deeply supercooled liquid and finally, very close to the plateau temperature at $T=243 \mathrm{~K}$. The arrows mark the $o$-Ps lifetimes at the corresponding characteristic PALS temperatures which indicate that a short-time part of the relaxation time distribution for the temperatures $185 \mathrm{~K}, 210 \mathrm{~K}$, and $243 \mathrm{~K}$ reach the typical time scale of the PALS method.

time, $\tau_{3}$, above $T_{g}^{\mathrm{PALS}}$ in Fig. 1. On increasing the temperature, the fraction of $\beta$ peak overcoming the ns scale increases and in between $210 \mathrm{~K}$ and $220 \mathrm{~K}$ also a short time tail of the $\alpha$ relaxation peak reaches the ns level. Further, regarding the secondary $\beta$ process contribution, Figure 6 shows both the width parameters of the Gaussian distribution function for the $\beta$ relaxation as obtained from the spectral analysis by using the WW approach as a function of temperature over the whole $T$ range of the existence of the $\beta_{\text {eff }}$ process [23]. A pronounced change in the width of the activation energy distribution, $\sigma_{E_{\beta}}$, and the corresponding width of the $\beta$ relaxation time distribution, $\sigma_{\log \tau_{\beta}}$, at around $210 \mathrm{~K}$ is evident. In Fig. 7 the distribution functions of the secondary relaxation times $G\left(\log _{10} \tau_{\beta}\right)$ vs $\log _{10} \tau_{\beta}$ for the $\beta$ process at several selected temperatures are displayed. As it can be seen, at $140 \mathrm{~K}$, i.e., in the deep glassy state, the $\beta$ relaxation time distribution is characterized by overall longer times compared to the PALS method in full consistency with Fig. 2. On increasing the temperature, $G\left(\log _{10} \tau_{\beta}\right)$ shifts to lower values and a very short time tail of the relaxation time distribution approaches the relevant PALS time scale already at around $T_{g}^{\text {PALS }}$. On further increasing the temperature, at $T_{b 1}$ a certain fraction of the $\beta_{\text {eff }}$ relaxation time distribution becomes shorter than the ns level as depicted by the arrows at the characteristic temperatures. These findings in Figs. 2, 6, and 7 correlate with the fact that the $\tau_{3}$ vs $T$ dependence increases more steeply with temperature above $T_{b 1}$.

All these empirical correlations between the effects on PALS response and the relaxation dynamics can be explained by using the general free volume idea that the $o$-Ps probe"senses" a part of the whole free volume distribution under 
appropriate free space and time conditions [7-10]. The former condition means that the $o$-Ps probe can sample local free volumes larger than the $o$-Ps probe size and the latter one means that the life of local free volume entities must be longer then the $o$-Ps lifetime. Thus, a part of the whole free volume distribution can be eliminated or blocked for detection by the $o$-Ps probe due to some motion which occurs on the appropriate time and amplitude scale. In other words, all the motions with the shorter time scale having the sufficient large displacements can contribute to the so-called "closing" part of the whole free volume distribution which thus becomes ineffective for the $o$-Ps probe sampling. Thus, in our case, the correlations between the PALS and BDS data in Figs. 2, 6, and 7 provide the evidence in favor of the blocking hypothesis of the PALS response in DEP. The highfrequency part of the distribution of the local $\beta_{\text {eff }}$ process as well as of the high-frequency tail of the $\alpha$ process occur on the shorter time scale compared to the observed $o$-Ps lifetime. Accordingly, the small size fraction of the free volume distribution becomes inaccessible for $o$-Ps localization due to these rapid motions of the sufficiently high frequency and amplitude. Consequently, the $o$-Ps probe has more chance to be localized and annihilated from the larger free volume holes resulting into the longer $o$-Ps lifetimes and the observed change of slope in the $\tau_{3}-T$ plot. Thus, the PALS method is able to measure the effective free volume situation in the condensed matter.

Finally, an interesting finding can be seen from comparison of the PALS response recorded in heating scans and the crossover phenomena in the parameters characterizing the $\beta_{\text {eff }}$ and $\alpha$ processes in Figs. 3-6. Decrease in the $\tau_{3}$ value just above $210 \mathrm{~K}$ down to a level lower than that for the glassy state and the following drastic increase above $270 \mathrm{~K}$ approaching the plateau level of the normal liquid are observed in this case. As the latter effect occurs very close to the melting temperature of the crystal form of DEP $T_{m}^{\mathrm{DSC}}$ $=273 \mathrm{~K}[35]$ it can be concluded that the structural transformation from deeply supercooled liquid to crystalline phase takes place, being responsible for the former effect. Then, although it is quite generally believed that the crystallization is controlled by the primary process, this finding seems to suggest that also the secondary process might be of relevance in the observed phase change in DEP, at least.

In summary, all these empirical facts point to a very close relationship between the PALS response and the dynamical features of the matrix, especially those of the high-frequency character, which at low temperatures are associated in majority with the secondary $\beta_{\text {eff }}$ process and in minority with the high-frequency part of the primary $\alpha$ relaxation. Thus, in PALS experiment we observe the effective PALS response of a system which is given by a close interplay of the corresponding time scales of the relevant annihilation and dynamic phenomena. The validity of this finding on a typical small molecular glass-forming compound should be further verified for other types of glass-forming systems including polymer ones. The works in this direction are in progress.

\section{CONCLUSIONS}

A combined analysis of the PALS and BDS databases on a small molecular glass-former system of diethyl phthalate has been performed. Comparison at phenomenological level revealed a number of coincidences between the various effects on the PALS response and various parameters of the dielectric response. In particular, the numerous changes in time and strength relaxation parameters of the secondary $\beta_{\text {eff }}$ process appear to coincide with the bend effects on the PALS response at $T_{b 1}$ and $T_{b 2}$, respectively. These findings point to the effective nature of the PALS response being influenced in majority by the local $\beta_{\text {eff }}$ process and in minority also by the high-frequency tail of the $\alpha$ process.

It also confirms that PALS is able to sense the dynamics of glass-forming systems.

\section{ACKNOWLEDGMENTS}

This work was supported by the VEGA Agency, Slovakia under Grants No. 2/6035/26. [to one of the authors (J.B.)] and No. 2/7121/27 [to one of the authors (O.S.)]. Two of the authors (A.A., J.C.) acknowledge the support by the Spanish Ministry of Science and Technology (MICyT) (Contract No. MAT 2004/01617) and by the Government of the Basque Country (Contract No. 9/UPV 00206.215-13568/2001).
[1] D. C. Champeney, R. B. N. Joarder, and J. C. Dore, Mol. Phys. 58, 337 (1986).

[2] B. Frick, D. Richter, and C. Ritter, Europhys. Lett. 9, 557 (1989).

[3] D. Morineau and C. Alba-Simionescu, J. Chem. Phys. 109, 8494 (1998).

[4] A. Narros, A. Arbe, F. Alvarez, J. Colmenero, R. Zorn, W. Schweika, and D. Richter, Macromolecules 38, 9847 (2005).

[5] J. D. Ferry, Viscoelastic Properties of Polymers, 3rd ed. (Wiley, New York, 1980).

[6] Diffusion in Polymers, edited by J. Crank and G. Park (Academic, New York, 1968).

[7] Principles and Application of Positron and Positronium Chemistry, edited by Y. C. Jean, P. E. Malton, and D. M. Schrader
(World Scientific, Singapore, 2003).

[8] J. Bartoš, in Encyclopedia of Analytical Chemistry, edited by R. A. Meyers (Wiley, Chichester, 2000), p. 7968.

[9] Y. C. Jean, Positron Spectroscopy of Solids (IOS, Ohmsha, Amsterdam, 1995), p. 563.

[10] S. Tao, J. Chem. Phys. 56, 5499 (1972); M. Eldrup, D. Lightbody, and J. N. Sherwood, Chem. Phys. 63, 51 (1981); H. Nakanishi, Y. C. Jean, and S. J. Wang, in Positron Annihilation Studies of Fluids, edited by S. C. Sharma (World Scientific, Singapore, 1988), p. 292.

[11] J. Bartoš and J. Krištiak, J. Phys. Chem. 104, 5666 (2000).

[12] P. Bandžuch, J. Krištiak, O. Šauša, and J. Zrubcová, Phys. Rev. B 61, 8784 ( 2000).

[13] J. Bartoš, J. Krištiak, O. Šauša, P. Bandžuch, and J. Zrubcová, 
Macromol. Symp. 158, 111 (2000).

[14] D. Bamford, G. Dlubek, A. Reiche, M. A. Alam, and W. Meyer, J. Chem. Phys. 113, 7260 (2001).

[15] G. Dlubek, E. M. Hassan, R. Krause-Rehberg, and J. Pionteck, Phys. Rev. E 73, 031803 (2006).

[16] J. Bartoš, O. Šauša, J. Krištiak, T. Blochowicz, and E. Rössler, J. Phys.: Condens. Matter 13, 11473 (2001).

[17] K. L. Ngai, L. R Bao, A. F. Yee, and C. L. Soles, Phys. Rev. Lett. 87, 215901 (2001).

[18] J. Bartoš, O. Šauša, P. Bandžuch, J. Zrubcová, and J. Krištiak, J. Non-Cryst. Solids 307-310, 417 (2002).

[19] J. Bartoš, O. Šauša, and J. Krištiak, in ARW NATO Series: Nonlinear Dielectric Phenomena in Complex Liquids, edited by S. J. Rzoska and V. Zhelezny (Kluwer Academic, Dordrecht, Netherlands, 2004), p. 289.

[20] J. Bartoš, D. Racko, O. Šauša, and J. Krištiak, in ARW NATO Series: Soft Matter under Exogenic Impacts. Fundamentals and Emerging Technologies, edited by S. J. Rzoska and V. Mazur (Springer-Verlag, Berlin, Germany, 2007), p. 110.

[21] A. Arbe, D. Richter, J. Colmenero, and B. Farago, Phys. Rev. E 54, 3853 (1996).

[22] P. Lunkenheimer, U. Schneider, R. Brand, and A. Loidl, Contemp. Phys. 41, 15 (2000).

[23] D. Gómez, A. Alegría, A. Arbe, and J. Colmenero, Macromolecules 34, 503 (2001).

[24] Broadband Dielectric Spectroscopy, edited by F. Kremer and A. Schönhals (Springer, Berlin, 2002).

[25] M. Tyagi, A. Alegría, and J. Colmenero, J. Chem. Phys. 122, 244909 (2005).

[26] R. A. Pethrick, F. M. Jacobsen, O. E. Mogensen, and M. Eldrup, J. Chem. Soc., Faraday Trans. 2 76, 225 (1980).

[27] Y. C. Jean, T. C. Sandreczki, and D. P. Ames, J. Polym. Sci.,
Part B: Polym. Phys. 24, 1247 (1986).

[28] J. R. Stevens, S. H. Chung, P. Horoyski, and K. R. Jeffrey, J. Non-Cryst. Solids 172-174, 1207 (1994).

[29] J. Bartoš, L. Andreozzi, M. Faetti, O. Šauša, D. Racko, and J. Krištiak, J. Non-Cryst. Solids 352, 4785 (2006).

[30] F. Stickel, Ph.D. thesis, Shaker, Aachen, 1995.

[31] A. Schönhals, Europhys. Lett. 56, 815 (2001).

[32] J. Bartoš, O. Šauša, D. Racko, J. Krištiak, and J. J. Fontanella, J. Non-Cryst. Solids 351, 2599 (2005).

[33] S. Pawlus, J. Bartoš, O. Šauša, J. Krištiak, and M. Paluch, J. Chem. Phys. 124, 104505 (2006).

[34] M. H. Cohen and G. S. Grest, Phys. Rev. B 20, 1077 (1979); G. S. Grest and M. H. Cohen, ibid. 21, 4113 (1980); Adv. Chem. Phys. 48, 455 (1981).

[35] S. S. N. Murthy, X. Gangasharan, and S. K. Nayak, J. Chem. Soc., Faraday Trans. 89, 509 (1993).

[36] J. Krištiak, J. Bartoš, K. Krištiaková, O. Šauša, and P. Bandžuch, Phys. Rev. B 49, 6601 (1994).

[37] P. Kirkegaard, M. Eldrup, O. E. Mogensen, and N. J. Pedersen, Comput. Phys. Commun. 23, 307 (1989).

[38] G. Williams and C. D. Watts, in NMR, Basic Principles and Progress, edited by P. Diehl, E. Fluck, and R. Kosfeld (Springer-Verlag, Berlin, 1971), Vol. 4, p. 271; G. Williams, Adv. Polym. Sci. 33, 60 (1979).

[39] F. Alvarez, A. Hoffman, A. Alegría, and J. Colmenero, J. Chem. Phys. 105, 432 (1996).

[40] C. Svanberg and R. Bergman, Philos. Mag. 87, 383 (2007).

[41] R. Bergman, F. Alvarez, A. Alegría, and J. Colmenero, J. Chem. Phys. 109, 7546 (1998).

[42] B. Malhotra and R. A. Pethrick, J. Chem. Soc., Faraday Trans. 2 78, 97 (1982). 